\title{
A PLASTIC SOCKET AND STUMP CASTING TECHNIQUE FOR ABOVE-KNEE PROSTHESES
}

\author{
J. A. E. Gleave, Hong Kong \\ Officer in Charge, Orthopaedic and Prosthetic Appliance Department, Hong Kong Government Medical Department
}

Above-knee artificial limbs with wooden or metal sockets have been produced successfully for many years and these materials will doubtless continue to be used in the future. Circumstances arise, however, when it is necessary to find an alternative material that has similar properties to wood or metal, but which may be used by technicians who do not possess the long experience of the wood or metal craftsman. Also in some countries conventional materials may be unsuitable by reason of climate or economy. There may also be places where prosthetic facilities are limited and where there are many patients needing prostheses which can be properly fitted economically and without delay.

There follows a description of the procedure evolved in this department to meet these needs.

\section{STUMP CASTS}

The prerequisite for the successful use of synthetic materials in socket manufacture is an accurate plaster cast of the stump; the cast must conform to the established criteria of socket shape and it should be taken under conditions of weight bearing. Plaster wrap casts have been found unsatisfactory because the positives had to be extensively modified.

Consequently a new technique of cast taking was evolved, and an adjustable socket and stand were designed (Figs. 1 and 2). This enabled a properly aligned cast to be taken under conditions of weight bearing, achieving an "ideal " shape, yet one which also conformed to individual stump contours.

The apparatus consists of the proximal three inches of a socket of an accepted shape, which is divided vertically into seven segments. These are linked together on a horizontal ring. The positions of two of the segments-the ischial seat and adductor channel-are fixed on the ring but they can be moved anteriorly and posteriorly by a screw adjustment. The other segments can move horizontally on the ring and can be locked in any desired position: they can be adjusted to the stump by a screw to which they are fixed by universal joints to allow alignment to the stump surface. This assembly is fixed on to a stand which allows adjustment for flexion, extension, height, medial or lateral rotation, and anterior or posterior displacement of the socket. There is also a detachable clamp which is used to hold the cast pipe vertically while pouring the plaster positive.

\section{CAST PROCEDURE}

The stump is carefully examined for the position of scar tissue and sensitive areas. Musculature, thickness of subcutaneous tissue and the ranges of stump movement are assessed. Measurements of the stump are made circumferentially at two-inch intervals, from the perineum to the distal end and from the greater trochanter to the distal end of the femoral remnant. The dimensions of the sound limb are also measured.

A three-point caliper is used to obtain a triangulation between the ischial tuberosity, the adductor tendon and the greater trochanter. These dimensions are transferred to the appropriate segments of the socket which are adjusted accordingly, and the stand is adjusted to the approximate height. After an application of vaseline to the patient's stump he places it in the socket and is asked to bear weight. The height of the socket and the remaining segments are adjusted around the stump to give a correct fit-taking into account any flexion, extension or other requirements-and the apparatus is then locked into position (Fig. 3). 


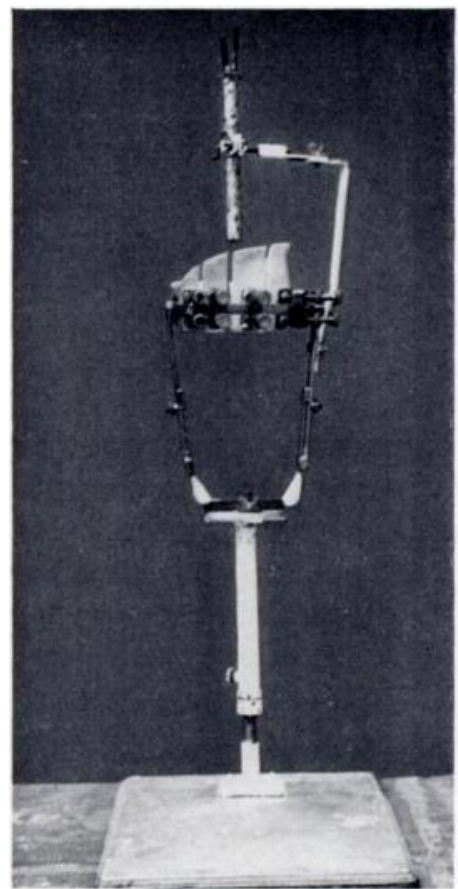

Fig. 1

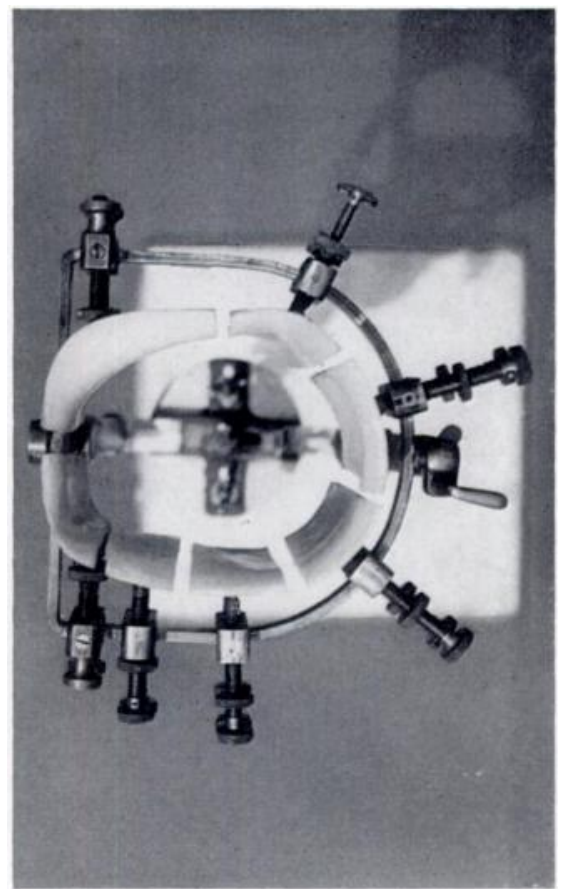

FiG. 2

Figure 1-The cast-taking stand assembled with cast pipe alignment fixture in position. Figure 2-A view of the socket from above to show the division of the socket segments, the ring and the screw adjustments.

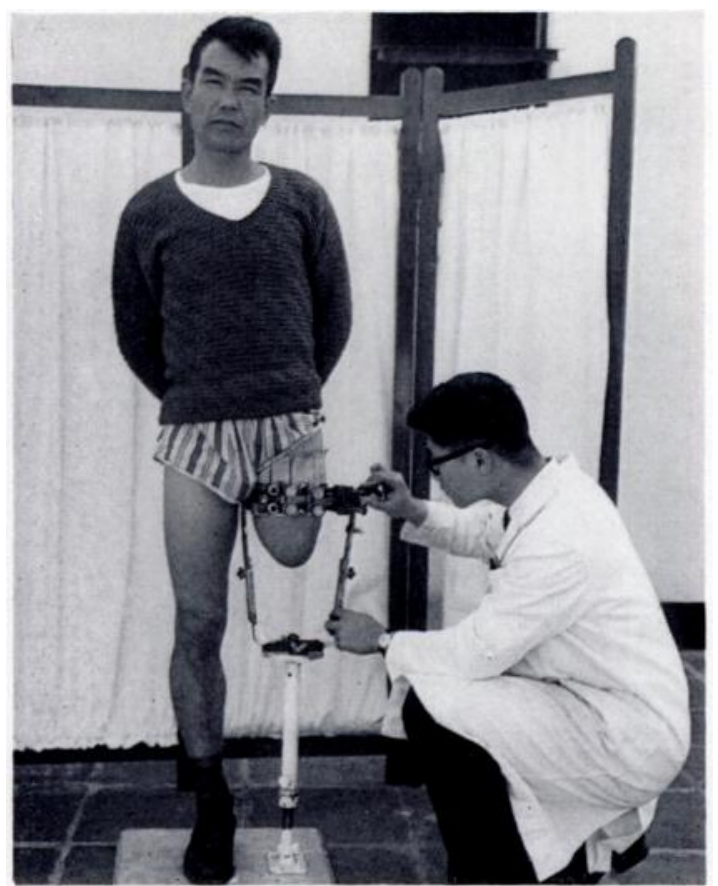

FiG. 3

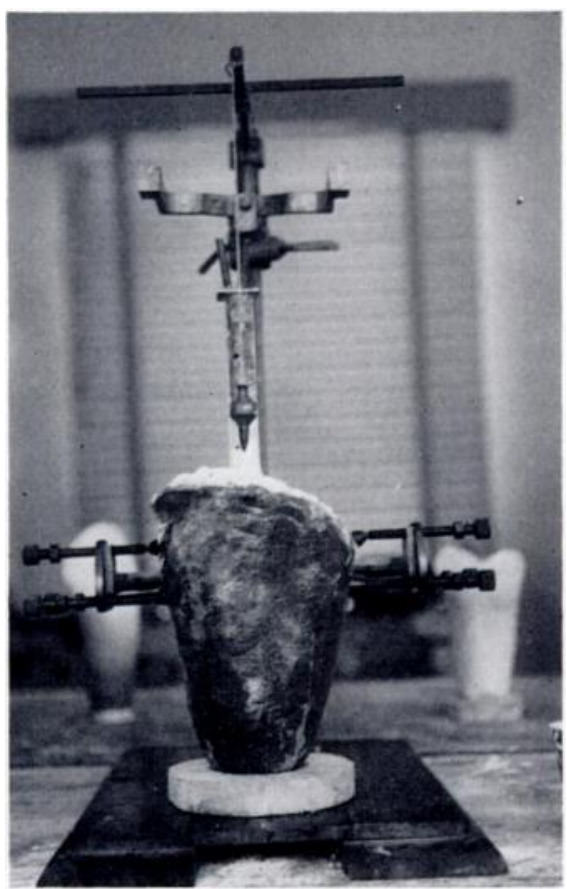

Fig. 4

Figure 3-The socket segments being adjusted on a patient. Figure 4-Alignment of the completed socket on its wooden base.

VOI. 47 B, NO. 1, FEBRUAR.Y 1965 
Next, a plaster bandage is wrapped round that part of the stump protruding below the edge of the socket; the upper edge of the bandage should include at least half an inch of the distal circumference of the socket. While the plaster is setting, the technician applies manual pressure over the lateral aspect of the stump. When the plaster has hardened, the patient gently removes his stump from the socket.

It is desirable to have a hollow cast for the socket production in order to facilitate subsequent breaking out of the cast after moulding. Thick plaster is applied with a spatula around the inner wall of the cast socket to a thickness of about half an inch. The cast pipe is then mounted on the stand and aligned vertically into the cast and paper is packed lightly around it to within half an inch of the top of the plaster. More plaster is applied to build up the cast to at least one inch above the top of the socket rim.

When the plaster has hardened, the cast is removed from the apparatus and is suitably dressed and smoothed according to the measurements and observations made at the initial examination. Casts produced by this method usually require little modification because the adjustable socket gives an accurate guide to shape, and all that remains to be done is to smooth the surface to give correct pressures over the stump surface and, if necessary, alter the height of the socket brim or the angle of the ischial seat if the stump is short and flexed.

\section{SOCKET}

The actual socket material must meet certain demands. These are economy, durability, lightness and non-toxicity; also it must be capable of local adjustment either by grinding or local heating. Numerous materials were tried experimentally and rejected, including polyester resin and glass fibre, urea formaldehyde resinated felt (Durestos), rigid polyisocyanate foam, epoxide resin and nylon felt. Finally a material was found which met all the requirements outlined above; this was a composite material consisting of epoxide resin, ground cork and glass-fibre needleloom mat.

Construction-A sheath of polyvinylchloride foil is drawn tightly over the cast, trimmed at the distal end and the open end sealed with a cap of the same material. The air is then evacuated from the inside of the sheath, which is now a smooth impervious covering preventing water or water vapour from the cast from inhibiting the curing of the resin, and also acting as a release agent. A second, larger, polyvinylchloride cone is prepared and loosely drawn over the cast. Catalysed epoxide resin and cork powder are mixed together in the proportion of four to five by weight, and the mixture is poured into the open end of the outer sheath and kneaded around the cast until a thickness of about half an inch is obtained all round. Both ends of the outer sheath are tied off and a vacuum is applied in order to give a homogeneous structure free from air bubbles.

When the socket has hardened, the outer sheath is carefully drawn off and the surface is roughened and covered with needleloom glass-fibre mat (one ounce per square foot) over which resin is stippled with a brush. The outer sheath is replaced and the vacuum reapplied. When this second application of resin has set, the socket is placed in an air circulating oven and cured for two hours at 80 degrees Centigrade. The outer sheath is then removed and the surface of the socket roughened. A piece of seasoned wood half an inch thick and slightly larger in diameter than the intended thigh section is placed upon a horizontal surface. With the cast pipe aligned vertically the socket is bonded to the wood with resin (Fig. 4). When this has set the contours of the socket are faired in with resin and cork. For this operation coarser grains of cork may be used than for making the socket. Other materials such as polyurethane or rigid polyisocyanate foams may also be used.

The plaster cast is broken out, the socket trimmed and set upon an adjustable leg or trial prosthesis. It is important, however, that the surface to which the socket is fitted should be horizontal; if not the alignment obtained in the initial cast taking and maintained through socket fabrication will not be reproduced. 


\section{OBSERVATIONS}

With this form of socket fabrication two important points arise. In its original concept it was intended that the socket should combine two ideal forms of fitting: first, that the interior of the socket should fit the stump with a firm overall contact; and second, that because individual stumps vary in length, shape, musculature and depth of subcutaneous tissue, the shape of the socket should conform to the individual contours of the stump. In the first it was found that the overall contact of a socket gave the amputee a greater feeling of stability and control with the prosthesis. This was important because many of these patients had little or no pre-prosthetic training. No instances of stump oedema have been observed nor has excessive sweating been noted.

In the second point the adaption of the socket shape to suit the individual stump contour gave rise to study. It was found that-apart from the fixed ischial seat and adductor channel-the shape of the antero-postero-lateral portion of each socket differed with each individual, thus being somewhat at variance with accepted "quadrilateral " or " anatomical " shapes at present in general use. This may possibly be because a socket of conventional shape conforms to methods of mass production although they may need modification at fitting. With this method of socket production modifications are carried out before the initial fitting and usually only one or two fittings are needed before completing the limb.

When a patient received some post-prosthetic training retention of the limb could be achieved by muscle action, but largely because of the social and economic conditions in this area, prosthetic training was not given as often as desirable. For this reason a light flexible pelvic suspension was fitted to all prostheses. Hip joints have only been found necessary with very short flexed stumps.

In this experiment it was thought that the production method would be quickly mastered by inexperienced staff because the manipulation of the materials was relatively simple. The technique of socket production can be mastered by an artisan with as little as two days' instruction. The technique of stump analysis and cast modification requires a little longer training, usually about a week.

The entire process, including measurement and cast-taking, takes about five hours. Socket modifications during fitting are usually slight and are easily done by grinding or local heating.

During the past two and a half years 132 of these limbs have been supplied; of these, nine have returned for refitting because of stump atrophy. One patient showed signs of allergy to the socket material, though this was doubtful because $\mathrm{pH}$ tests on the socket surface were neutral and patch tests on the patient were inconclusive. The remainder appeared to be satisfactory.

It is not suggested that the materials and methods described should replace the use of wood or metal, for these materials have their established place in prosthetic fabrication. It is, however, a useful economic alternative, perhaps with certain advantages, especially in those areas where there are few skilled prosthetic technicians. It is appreciated that there are at present under study similar methods of preparing stump sockets, utilising casting frames and synthetic materials; but the simplified techniques developed in Hong Kong seem worthy of publication.

\section{SUMMARY}

1. This is a report on the production of sockets for above-knee prostheses utilising a composite laminate of synthetic and natural materials and using a principle of total stump contact with the socket, which before fitting is shaped to suit individual stump contours.

2. A new method of taking casts is described, as is the production of the socket, and observations on the use of the prosthesis over the last two and a half years are made.

I wish to thank Dr D. J. M. MacKenzie, Director of Medical and Health Services, Hong Kong, for permission to publish this paper.

Vol.. 47 B, NO. I, FEBRUARY 1965 